\title{
COHOMOLOGICAL INVARIANTS AND R-TRIVIALITY OF ADJOINT CLASSICAL GROUPS
}

\author{
GRÉGORY BERHUY, MARINA MONSURRÒ, AND JEAN-PIERRE TIGNOL
}

\begin{abstract}
Using a cohomological obstruction, we construct examples of absolutely simple adjoint classical groups of type ${ }^{2} A_{n}$ with $n \equiv 3 \bmod 4, C_{n}$ or ${ }^{1} D_{n}$ with $n \equiv 0 \bmod 4$, which are not $R$-trivial hence not stably rational.
\end{abstract}

\section{INTRODUCTION}

For an algebraic group $G$ defined over a field $F$, let $G(F) / R$ be the group of $R$-equivalence classes introduced by Manin in [6]. The algebraic group $G$ is called $R$-trivial if $G(L) / R=1$ for every field extension $L / F$. It was established by ColliotThélène and Sansuc in [2] (see also [7, Proposition 1]) that the group $G$ is $R$-trivial if the variety of $G$ is stably rational.

In this paper, we focus on the case where $G$ is an absolutely simple classical group of adjoint type. Adjoint groups of type ${ }^{1} A_{n}$ or $B_{n}$ are easily seen to be rational (see [7, pp. 199, 200]). Voskresenskiu and Klyachko [11, Cor. of Th. 8] proved that adjoint groups of type ${ }^{2} A_{n}$ are rational if $n$ is even, and Merkurjev [7, Prop. 4] showed that adjoint groups of type $C_{n}$ are stably rational for $n$ odd. On the other hand, Merkurjev also produced in [7] examples of adjoint groups of type ${ }^{2} A_{3}\left(={ }^{2} D_{3}\right)$ and of type ${ }^{2} D_{n}$ for any $n \geq 4$ which are not $R$-trivial, hence not stably rational. Examples of adjoint groups of type ${ }^{1} D_{4}$ which are not $R$-trivial were constructed by Gille in [3].

The goal of the present paper is to construct examples of adjoint groups of type ${ }^{2} A_{n}$ with $n \equiv 3 \bmod 4$ and of adjoint groups of type $C_{n}$ or ${ }^{1} D_{n}$ with $n \equiv 0 \bmod 4$ which are not $R$-trivial. Our constructions are based on Merkurjev's computation in [7] of the group of $R$-equivalence classes of adjoint classical groups, which we now recall briefly. According to Weil (see $[4, \S 26]$ ), every absolutely simple classical group of adjoint type over a field $F$ of characteristic different from 2 can be obtained as the connected component of the identity in the automorphism group of a central ${ }^{1}$ simple algebra with involution $(A, \sigma)$ over $F$. Let $\operatorname{Sim}(A, \sigma)$ be the algebraic group of similitudes of $(A, \sigma)$, defined (as a group scheme) by

$$
\operatorname{Sim}(A, \sigma)(E)=\left\{u \in A \otimes_{F} E \mid(\sigma \otimes \operatorname{Id})(u) u \in E^{\times}\right\}
$$

2000 Mathematics Subject Classification. 11E57, 20G15.

The authors gratefully acknowledge support from the TMR network " $K$-theory and linear algebraic groups" (ERB FMRX CT97-0107). The third author is also grateful to the National Fund for Scientific Research (Belgium) for partial support.

${ }^{1}$ We use the same terminology as in [4]. In particular, the center of $A$ is $F$ if $\sigma$ is of the first kind; it is a quadratic étale extension of $F$ if $\sigma$ is of the second kind. 
for every commutative $F$-algebra $E$, and let $\operatorname{PSim}(A, \sigma)$ be the group of projective similitudes,

$$
\operatorname{PSim}(A, \sigma)=\operatorname{Sim}(A, \sigma) / R_{K / F}\left(\mathbf{G}_{\mathrm{m}, K}\right)
$$

where $K$ is the center of $A$. The connected component of the identity in these groups is denoted by $\operatorname{Sim}^{+}(A, \sigma)$ and $\mathbf{P S i m}^{+}(A, \sigma)$ respectively. We let $\operatorname{Sim}(A, \sigma)$, $\operatorname{PSim}(A, \sigma), \operatorname{Sim}^{+}(A, \sigma)$ and $\operatorname{PSim}^{+}(A, \sigma)$ denote the corresponding groups of $F$ rational points:

$$
\operatorname{Sim}(A, \sigma)=\operatorname{Sim}(A, \sigma)(F), \quad \operatorname{PSim}(A, \sigma)=\operatorname{PSim}(A, \sigma)(F), \quad \text { etc. }
$$

The group $\operatorname{PSim}^{+}(A, \sigma)$ is canonically isomorphic (under the map which carries every similitude $g$ to the induced inner automorphism $\operatorname{Int}(g))$ to the connected component of the identity in the automorphism group of $(A, \sigma)$. To describe the group of $R$-equivalence classes of $\operatorname{PSim}^{+}(A, \sigma)$, consider the homomorphism

$$
\mu: \operatorname{Sim}(A, \sigma) \rightarrow \mathbf{G}_{\mathrm{m}}
$$

which carries every similitude to its multiplier

$$
\mu(g)=\sigma(g) g .
$$

Let $G^{+}(A, \sigma)=\mu\left(\operatorname{Sim}^{+}(A, \sigma)\right) \subset F^{\times}$and $N K^{\times}=\mu\left(K^{\times}\right) \subset F^{\times}$(so $N K^{\times}=F^{\times 2}$ if $K=F)$. Let also $\operatorname{Hyp}(A, \sigma)$ be the subgroup of $F^{\times}$generated by the norms of the finite extensions $L$ of $F$ such that $(A, \sigma)$ becomes hyperbolic after scalar extension to $L$. In [7, Theorem 1], Merkurjev shows that the multiplier map $\mu$ induces a canonical isomorphism

$$
\operatorname{PSim}^{+}(A, \sigma) / R \simeq G^{+}(A, \sigma) /\left(N K^{\times} \cdot \operatorname{Hyp}(A, \sigma)\right) .
$$

For any positive integer $d$, let $H^{d}\left(F, \mu_{2}\right)$ be the degree $d$ cohomology group of the absolute Galois group of $F$ with coefficients $\mu_{2}=\{ \pm 1\}$. In Section 3 we consider the case where $\sigma$ is of the first kind. If it is orthogonal, we assume further that its discriminant is trivial. Assuming the index of $A$ divides $\frac{1}{2} \operatorname{deg} A$, we construct a homomorphism

$$
\Theta_{1}: \operatorname{PSim}^{+}(A, \sigma) / R \rightarrow H^{4}\left(F, \mu_{2}\right),
$$

and give examples where this homomorphism is nonzero, hence $\operatorname{PSim}^{+}(A, \sigma) / R \neq 1$. Similarly, if $\sigma$ is of the second kind and the exponent of $A$ divides $\frac{1}{2} \operatorname{deg} A$, we construct in Section 4 a homomorphism

$$
\Theta_{2}: \operatorname{PSim}^{+}(A, \sigma) / R \rightarrow H^{3}\left(F, \mu_{2}\right)
$$

and show that this map is nonzero in certain cases. In all the examples where we show $\Theta_{1} \neq 0$ or $\Theta_{2} \neq 0$, the algebra with involution has the form $(A, \sigma)=(B, \rho) \otimes$ $(C, \tau)$ where $\rho$ is an orthogonal involution which admits improper similitudes.

Throughout the paper, the characteristic of the base field $F$ is different from 2 .

\section{IMPROPER SIMILITUDES}

Let $(A, \sigma)$ be a central simple $F$-algebra with orthogonal involution of degree $n=2 m$. The group of similitudes $\operatorname{Sim}(A, \sigma)$ is denoted $\mathbf{G O}(A, \sigma)$. This group is not connected. Its connected component of the identity $\mathbf{G O}^{+}(A, \sigma)$ is defined by the equation

$$
\operatorname{Nrd}_{A}(g)=\mu(g)^{m}
$$


where $\operatorname{Nrd}_{A}$ is the reduced norm. We denote by $\operatorname{GO}(A, \sigma)$ and $\mathrm{GO}^{+}(A, \sigma)$ the group of $F$-rational points

$$
\operatorname{GO}(A, \sigma)=\mathbf{G O}(A, \sigma)(F), \quad \mathrm{GO}^{+}(A, \sigma)=\mathbf{G O}^{+}(A, \sigma)(F) .
$$

The elements in $\mathrm{GO}^{+}(A, \sigma)$ are called proper similitudes, and those in the nontrivial coset

$$
\mathrm{GO}^{-}(A, \sigma)=\left\{g \in \mathrm{GO}(A, \sigma) \mid \operatorname{Nrd}_{A}(g)=-\mu(g)^{m}\right\}
$$

are called improper similitudes.

For example, if $m=1$ (i.e. $A$ is a quaternion algebra), then every orthogonal involution has the form $\sigma=\operatorname{Int}(q) \circ \gamma$, where $\gamma$ is the canonical involution, $q$ is an invertible pure quaternion and $\operatorname{Int}(q)$ is the inner automorphism induced by $q$, mapping $x \in A$ to $q x q^{-1}$. It is easily checked that

$$
\mathrm{GO}^{+}(A, \sigma)=F(q)^{\times} \quad \text { and } \quad \mathrm{GO}^{-}(A, \sigma)=q^{\prime} F(q)^{\times},
$$

where $q^{\prime}$ is a unit which anticommutes with $q$. Therefore, $\mathrm{GO}^{-}(A, \sigma) \neq \varnothing$.

If $m>1$, the existence of improper similitudes is an important restriction on $A$ and $\sigma$, since it implies that $A$ is split by the quadratic étale $F$-algebra $F[\sqrt{\operatorname{disc} \sigma}]$, where disc $\sigma$ is the discriminant of $\sigma$, see [9, Theorem A] or [4, (13.38)]. In particular, the index of $A$ satisfies ind $A \leq 2$, i.e. $A$ is Brauer-equivalent to a quaternion algebra. Moreover, if $m$ is even, then $-1 \in \operatorname{Nrd}_{A}(A)$, see [9, Corollary 1.13]. There is no other restriction on $A$, as the following proposition shows.

1. Proposition. Let $H$ be an arbitrary quaternion $F$-algebra and let $m$ be an arbitrary integer. If $m$ is even, assume $-1 \in \operatorname{Nrd}_{H}\left(H^{\times}\right)$. Then the algebra $M_{m}(H)$ carries an orthogonal involution which admits improper similitudes.

Proof. Suppose first $m$ is odd. Let $i, j$ be elements in a standard quaternion basis of $H$. We set

$$
\sigma=t \otimes(\operatorname{Int}(i) \circ \gamma) \quad \text { on } M_{m}(H)=M_{m}(F) \otimes_{F} H,
$$

where $\gamma$ is the canonical involution on $H$. It is readily verified that $1 \otimes j$ is an improper similitude of $\sigma$.

Suppose next $m$ is even, and $q \in H$ satisfies $\operatorname{Nrd}_{H}(q)=-1$. We pick a quaternion basis $1, i, j, k=i j$ such that $i$ commutes with $q$, and set

$$
\sigma=\operatorname{Int} \operatorname{diag}(j, i, \ldots, i) \circ(t \otimes \gamma) \quad \text { and } \quad g=\operatorname{diag}(j, q j, \ldots, q j) .
$$

Again, computation shows that $g$ is an improper similitude of $\sigma$.

Necessary and sufficient conditions for the existence of improper similitudes for a given involution $\sigma$ are not known if $m \geq 4$. For $m=2$ (resp. $m=3$ ), Corollary (15.9) (resp. (15.26)) in [4] shows that $\mathrm{GO}^{-}(A, \sigma) \neq \varnothing$ if and only if the Clifford algebra $C(A, \sigma)$ has outer automorphisms (resp. outer automorphisms which commute with its canonical involution). (For $m=2$ another equivalent condition is that $A$ is split by the center of $C(A, \sigma)$, see [4, (15.11)] or [9, Prop. 1.15].) We use this fact to prove the following result:

2. Proposition. Let $(A, \sigma)$ be a central simple $F$-algebra with orthogonal involution of degree 4. Assume that $A$ is not split and $\operatorname{disc} \sigma \neq 1$. Then there exists a field extension $L / F$ such that $A_{L}$ is not split and $\mathrm{GO}^{-}\left(A_{L}, \sigma_{L}\right) \neq \varnothing$. 
Proof. By hypothesis, $F(\sqrt{\operatorname{disc} \sigma})$ is a quadratic field extension of $F$. We denote it by $K$ for simplicity and let $\iota$ be its nontrivial $F$-automorphism. The Clifford algebra $C=C(A, \sigma)$ is a quaternion $K$-algebra. Let $X$ be the Severi-Brauer variety of $C \otimes{ }_{K}{ }^{\iota} C$ and let $L$ be the function field of its Weil transfer:

$$
L=F\left(R_{K / F}(X)\right) .
$$

Then $\left(C \otimes_{K}{ }^{\iota} C\right) \otimes_{K} K L$ splits, so $C_{K L}$ is isomorphic to ${ }^{\iota} C_{K L}$, which means that $C_{K L}$ has outer automorphisms. By $[4,(15.9)]$, it follows that $\mathrm{GO}^{-}\left(A_{L}, \sigma_{L}\right) \neq \varnothing$.

On the other hand, by [9, Corollary 2.12], the kernel of the scalar extension map $\operatorname{Br}(F) \rightarrow \operatorname{Br}(L)$ is generated by the corestriction of $C \otimes{ }_{K}{ }^{\iota} C$. Since this corestriction is trivial, $A_{L}$ is not split.

\section{TRACE FORMS}

In this section, $A$ is a central simple $F$-algebra of even degree with an involution $\sigma$ of the first kind. We consider the quadratic forms $T_{A}$ and $T_{\sigma}$ on $A$ defined by

$$
T_{A}(x)=\operatorname{Trd}_{A}\left(x^{2}\right), \quad T_{\sigma}(x)=\operatorname{Trd}_{A}(\sigma(x) x) \quad \text { for } x \in A,
$$

where $\operatorname{Trd}_{A}$ is the reduced trace on $A$. We denote by $T_{\sigma}^{+}$(resp. $T_{\sigma}^{-}$) the restriction of $T_{\sigma}$ to the space $\operatorname{Sym}(\sigma)$ of symmetric elements (resp. to the space $\operatorname{Skew}(\sigma)$ of skew-symmetric elements), so that

$$
T_{A}=T_{\sigma}^{+} \perp-T_{\sigma}^{-} \quad \text { and } \quad T_{\sigma}=T_{\sigma}^{+} \perp T_{\sigma}^{-} .
$$

Recall that if $\sigma$ is orthogonal the (signed) discriminant $\operatorname{disc} T_{\sigma}^{+}$is equal to the discriminant $\operatorname{disc} \sigma$ up to a factor which depends only on the degree of $A$, see for instance $[4,(11.5)]$. In the following, we denote by $I^{n} F$ the $n$-th power of the fundamental ideal $I F$ of the Witt ring $W F$.

3. Lemma. Let $\sigma, \sigma_{0}$ be two involutions of the first kind on $A$.

- If $\sigma$ and $\sigma_{0}$ are both symplectic, then $T_{\sigma}^{+}-T_{\sigma_{0}}^{+} \in I^{3} F$.

- If $\sigma$ and $\sigma_{0}$ are both orthogonal, then $\operatorname{disc}\left(T_{\sigma}^{+}-T_{\sigma_{0}}^{+}\right)=\operatorname{disc} \sigma \operatorname{disc} \sigma_{0}$. Moreover, if $\operatorname{disc} \sigma=\operatorname{disc} \sigma_{0}$, then $T_{\sigma}^{+}-T_{\sigma_{0}}^{+} \in I^{3} F$.

Proof. The symplectic case has been considered in [1, Theorem 4]. For the rest of the proof, we assume that $\sigma$ and $\sigma_{0}$ are both orthogonal. By [4, (11.5)], there is a factor $c \in F^{\times}$such that

$$
\operatorname{disc} T_{\sigma}^{+}=c \operatorname{disc} \sigma \quad \text { and } \quad \operatorname{disc} T_{\sigma_{0}}^{+}=c \operatorname{disc} \sigma_{0}
$$

hence

$$
\operatorname{disc}\left(T_{\sigma}^{+}-T_{\sigma_{0}}^{+}\right)=\operatorname{disc} T_{\sigma}^{+} \operatorname{disc} T_{\sigma_{0}}^{+}=\operatorname{disc} \sigma \operatorname{disc} \sigma_{0} .
$$

To complete the proof, observe that the Witt-Clifford invariant $e_{2}\left(T_{\sigma}^{+}\right)$(or, equivalently, the Hasse invariant $w_{2}\left(T_{\sigma}^{+}\right)$) depends only on $\operatorname{disc} \sigma$ and on the Brauer class of $A$, as was shown by Quéguiner [10, p. 307]. Therefore, if $\operatorname{disc} \sigma=\operatorname{disc} \sigma_{0}$, then $e_{2}\left(T_{\sigma}^{+}\right)=e_{2}\left(T_{\sigma_{0}}^{+}\right)$, hence $T_{\sigma}^{+}-T_{\sigma_{0}}^{+} \in I^{3} F$ by a theorem of Merkurjev.

We next compute the Arason invariant $e_{3}\left(T_{\sigma}^{+}-T_{\sigma_{0}}^{+}\right) \in H^{3}\left(F, \mu_{2}\right)$ in the special case where $\sigma$ and $\sigma_{0}$ decompose. We use the following notation: $[A] \in H^{2}\left(F, \mu_{2}\right)$ is the cohomology class corresponding to the Brauer class of $A$ under the canonical isomorphism $H^{2}\left(F, \mu_{2}\right)={ }_{2} \operatorname{Br}(F)$. For $a \in F^{\times}$we denote by $(a)$ the cohomology class corresponding to the square class of $a$ under the canonical isomorphism $H^{1}\left(F, \mu_{2}\right)=F^{\times} / F^{\times 2}$. 
4. Lemma. Suppose $A=B \otimes_{F} C$ for some central simple F-algebras $B, C$ of even degree. Let $\rho$ and $\rho_{0}$ be orthogonal involutions on $B$ and let $\tau$ be an involution of the first kind on $C$. Let also $\sigma=\rho \otimes \tau$ and $\sigma_{0}=\rho_{0} \otimes \tau$.

If $\tau$ (hence also $\sigma$ and $\sigma_{0}$ ) is symplectic, then

$$
e_{3}\left(T_{\sigma}^{+}-T_{\sigma_{0}}^{+}\right)= \begin{cases}0 & \text { if } \operatorname{deg} C \equiv 0 \bmod 4, \\ \left(\operatorname{disc} \rho \operatorname{disc} \rho_{0}\right) \cup[C] & \text { if } \operatorname{deg} C \equiv 2 \bmod 4 .\end{cases}
$$

If $\tau$ (hence also $\sigma$ and $\sigma_{0}$ ) is orthogonal, then

$$
e_{3}\left(T_{\sigma}^{+}-T_{\sigma_{0}}^{+}\right)= \begin{cases}\left(\operatorname{disc} \rho \operatorname{disc} \rho_{0}\right) \cup(\operatorname{disc} \tau) \cup(-1) & \text { if } \operatorname{deg} C \equiv 0 \bmod 4, \\ \left(\operatorname{disc} \rho \operatorname{disc} \rho_{0}\right) \cup((\operatorname{disc} \tau) \cup(-1)+[C]) & \text { if } \operatorname{deg} C \equiv 2 \bmod 4 .\end{cases}
$$

Proof. The decomposition

$$
\operatorname{Sym}(\sigma)=(\operatorname{Sym}(\rho) \otimes \operatorname{Sym}(\tau)) \oplus(\operatorname{Skew}(\rho) \otimes \operatorname{Skew}(\tau))
$$

yields

$$
T_{\sigma}^{+}=T_{\rho}^{+} T_{\tau}^{+}+T_{\rho}^{-} T_{\tau}^{-} \quad \text { in } W F .
$$

Since $T_{B}=T_{\rho}^{+}-T_{\rho}^{-}$we may eliminate $T_{\rho}^{-}$in the equation above to obtain

$$
T_{\sigma}^{+}=T_{\rho}^{+} T_{\tau}^{+}+\left(T_{\rho}^{+}-T_{B}\right) T_{\tau}^{-} .
$$

Similarly,

$$
T_{\sigma_{0}}^{+}=T_{\rho_{0}}^{+} T_{\tau}^{+}+\left(T_{\rho_{0}}^{+}-T_{B}\right) T_{\tau}^{-}
$$

and subtracting the two equalities yields

$$
T_{\sigma}^{+}-T_{\sigma_{0}}^{+}=\left(T_{\rho}^{+}-T_{\rho_{0}}^{+}\right) T_{\tau}^{+}+\left(T_{\rho}^{+}-T_{\rho_{0}}^{+}\right) T_{\tau}^{-}=\left(T_{\rho}^{+}-T_{\rho_{0}}^{+}\right) T_{\tau} .
$$

Since $\operatorname{deg} C$ is even, we have $T_{\tau} \in I^{2} F$ (see $[4,(11.5)]$ ), hence

$$
e_{3}\left(T_{\sigma}^{+}-T_{\sigma_{0}}^{+}\right)=\left(\operatorname{disc}\left(T_{\rho}^{+}-T_{\rho_{0}}^{+}\right)\right) \cup e_{2}\left(T_{\tau}\right) \quad \text { in } H^{3}\left(F, \mu_{2}\right) .
$$

By Lemma 3 we have

$$
\operatorname{disc}\left(T_{\rho}^{+}-T_{\rho_{0}}^{+}\right)=\operatorname{disc} \rho \operatorname{disc} \rho_{0} .
$$

The computation of $e_{2}\left(T_{\tau}\right)$ in [10, Theorem 1] or [5] completes the proof.

Remark. If $\sigma$ and $\sigma_{0}$ are symplectic, the Arason invariant $e_{3}\left(T_{\sigma}^{+}-T_{\sigma_{0}}^{+}\right)$is the discriminant $\Delta_{\sigma_{0}}(\sigma)$ investigated in [1].

\section{INVOLUTIONS OF THE FIRST KIND}

In this section, $A$ is a central simple $F$-algebra of even degree, and $\sigma$ is an involution of the first kind on $A$. We assume ind $A$ divides $\frac{1}{2} \operatorname{deg} A$, i.e. $A \simeq M_{2}\left(A_{0}\right)$ for some central simple $F$-algebra $A_{0}$, so that $A$ carries a hyperbolic involution $\sigma_{0}$ of the same type as $\sigma$. If $\sigma$ is orthogonal, we assume $\operatorname{disc} \sigma=1\left(=\operatorname{disc} \sigma_{0}\right)$, so that in all cases $T_{\sigma}^{+}-T_{\sigma_{0}}^{+} \in I^{3} F$, by Lemma 3 .

5. Proposition. The map $\theta_{1}: \operatorname{Sim}(A, \sigma) \rightarrow H^{4}\left(F, \mu_{2}\right)$ defined by

$$
\theta_{1}(g)=(\mu(g)) \cup e_{3}\left(T_{\sigma}^{+}-T_{\sigma_{0}}^{+}\right)
$$

induces a homomorphism

$$
\Theta_{1}: \operatorname{PSim}^{+}(A, \sigma) / R \rightarrow H^{4}\left(F, \mu_{2}\right) .
$$

Moreover, for all $g \in \operatorname{Sim}(A, \sigma)$, we have

$$
\theta_{1}(g) \cup(-1)=0 \quad \text { in } H^{5}\left(F, \mu_{2}\right) .
$$


Proof. In view of the isomorphism (1), it suffices to show that for every finite field extension $L / F$ such that $(A, \sigma) \otimes_{F} L$ is hyperbolic and for every $x \in L^{\times}$,

$$
\left(N_{L / F}(x)\right) \cup e_{3}\left(T_{\sigma}^{+}-T_{\sigma_{0}}^{+}\right)=0 \quad \text { in } H^{4}\left(F, \mu_{2}\right) .
$$

The projection formula yields

$$
\left(N_{L / F}(x)\right) \cup e_{3}\left(T_{\sigma}^{+}-T_{\sigma_{0}}^{+}\right)=\operatorname{cor}_{L / F}\left((x) \cup e_{3}\left(T_{\sigma}^{+}-T_{\sigma_{0}}^{+}\right)_{L}\right) .
$$

Since $\sigma_{L}$ is hyperbolic, the involutions $\sigma_{L}$ and $\left(\sigma_{0}\right)_{L}$ are conjugate, hence

$$
e_{3}\left(T_{\sigma}^{+}-T_{\sigma_{0}}^{+}\right)_{L}=0 \text {. }
$$

For the last equality, observe that (2) yields the following equations in $W F$ :

$$
T_{\sigma}+T_{A}=\langle 1,1\rangle T_{\sigma}^{+} \quad \text { and } \quad T_{\sigma_{0}}+T_{A}=\langle 1,1\rangle T_{\sigma_{0}}^{+},
$$

hence

$$
T_{\sigma}-T_{\sigma_{0}}=\langle 1,1\rangle\left(T_{\sigma}^{+}-T_{\sigma_{0}}^{+}\right) .
$$

Since $\sigma_{0}$ is hyperbolic, we have $T_{\sigma_{0}}=0$. Moreover, for $g \in \operatorname{Sim}(A, \sigma)$ the map $x \mapsto g x$ is a similitude of $T_{\sigma}$ with multiplier $\mu(g)$, hence

$$
\langle 1,-\mu(g)\rangle T_{\sigma}=\langle 1,-\mu(g)\rangle\langle 1,1\rangle\left(T_{\sigma}^{+}-T_{\sigma_{0}}^{+}\right)=0 .
$$

Since

$$
e_{5}\left(\langle 1,-\mu(g)\rangle\langle 1,1\rangle\left(T_{\sigma}^{+}-T_{\sigma_{0}}^{+}\right)\right)=\theta_{1}(g) \cup(-1),
$$

the proposition follows.

6. Proposition. Let $(A, \sigma)=(B, \rho) \otimes(C, \tau)$, where $B$ and $C$ are central simple $F$-algebras of even degree and $\rho, \tau$ are involutions of the first kind. Suppose ind $B$ divides $\frac{1}{2} \operatorname{deg} B$ and $\rho$ is orthogonal. For $g \in \mathrm{GO}^{-}(B, \rho)$, we have $g \otimes 1 \in \operatorname{Sim}^{+}(A, \sigma)$ and

$$
\theta_{1}(g \otimes 1)= \begin{cases}0 & \text { if } \operatorname{deg} C \equiv 0 \bmod 4, \\ {[B] \cup[C]} & \text { if } \operatorname{deg} C \equiv 2 \bmod 4 .\end{cases}
$$

Proof. For $g \in \operatorname{GO}(B, \rho)$, we have

$$
\sigma(g \otimes 1) g \otimes 1=\rho(g) g=\mu(g)
$$

and

so $g \otimes 1 \in \operatorname{Sim}^{+}(A, \sigma)$.

$$
\operatorname{Nrd}_{A}(g \otimes 1)=\operatorname{Nrd}_{B}(g)^{\operatorname{deg} C},
$$

Since ind $B$ divides $\frac{1}{2} \operatorname{deg} B$, we may find a hyperbolic orthogonal involution $\rho_{0}$ on $B$, and set $\sigma_{0}=\rho_{0} \otimes \tau$, a hyperbolic involution on $A$ of the same type as $\sigma$.

If $\tau$ is symplectic, Lemma 4 yields

$$
e_{3}\left(T_{\sigma}^{+}-T_{\sigma_{0}}^{+}\right)= \begin{cases}0 & \text { if } \operatorname{deg} C \equiv 0 \bmod 4, \\ (\operatorname{disc} \rho) \cup[C] & \text { if } \operatorname{deg} C \equiv 2 \bmod 4 .\end{cases}
$$

The proposition follows by taking the cup-product with $(\mu(g))$, since $(\mu(g)) \cup$ $(\operatorname{disc} \rho)=[B]$ by $[9$, Theorem A] (see also $[4,(13.38)])$.

Suppose next $\tau$ is orthogonal. By Lemma 4 ,

$$
e_{3}\left(T_{\sigma}^{+}-T_{\sigma_{0}}^{+}\right)= \begin{cases}(\operatorname{disc} \rho) \cup(\operatorname{disc} \tau) \cup(-1) & \text { if } \operatorname{deg} C \equiv 0 \bmod 4, \\ (\operatorname{disc} \rho) \cup((\operatorname{disc} \tau) \cup(-1)+[C]) & \text { if } \operatorname{deg} C \equiv 2 \bmod 4 .\end{cases}
$$


Using again the equation $(\mu(g)) \cup(\operatorname{disc} \rho)=[B]$ and taking into account the equation $(-1) \cup[B]=0$, which follows from [9, Corollary 1.13], we obtain the formula for $\theta_{1}(g \otimes 1)$.

Using Proposition 6 , it is easy to construct examples where $\theta_{1} \neq 0$. For these examples, the map $\Theta_{1}$ of Proposition 5 is not trivial, hence $\operatorname{PSim}^{+}(A, \sigma)$ is not $R$-trivial.

7. Corollary. Let $Q, H$ be quaternion $F$-algebras satisfying

$$
(-1) \cup[H]=0 \text { in } H^{3}\left(F, \mu_{2}\right) \quad \text { and } \quad[H] \cup[Q] \neq 0 \text { in } H^{4}\left(F, \mu_{2}\right) .
$$

Let $A=M_{2 r}(H) \otimes M_{s}(Q)$, where $r$ is arbitrary and $s$ is odd. Let $\rho$ be an orthogonal involution on $M_{2 r}(H)$ which admits improper similitudes (see Lemma 1), and let $\tau$ be any involution of the first kind on $M_{s}(Q)$. Then $\operatorname{PSim}^{+}(A, \rho \otimes \tau)$ is not R-trivial.

To obtain explicit examples, we may take for $F$ the field of rational fractions in four indeterminates $F=\mathbb{C}\left(x_{1}, y_{1}, y_{2}, y_{2}\right)$ and set $H=\left(x_{1}, y_{1}\right)_{F}, Q=\left(x_{2}, y_{2}\right)_{F}$. Note that the degree of $A$ can be any multiple of 8 and that the conditions on $Q$ and $H$ in Corollary 7 imply ind $A=4$. Indeed, if there is a quadratic extension of $F$ which splits $Q$ and $H$, then $[H] \cup[Q]$ is a multiple of $(-1) \cup[H]$.

Other examples can be obtained from Proposition 2.

8. Corollary. Let $(B, \rho)$ be a central simple algebra of degree 4 and index 2 with orthogonal involution of nontrivial discriminant over a field $F_{0}$. Let $F=F_{0}(x, y)$ be the field of rational fractions in two indeterminates $x, y$ over $F_{0}$, and let $(C, \tau)$ be a central simple F-algebra with involution of the first kind such that

$$
\operatorname{deg} C \equiv 2 \bmod 4 \quad \text { and } \quad[C]=(x) \cup(y) \in H^{2}\left(F, \mu_{2}\right) .
$$

Then $\operatorname{PSim}^{+}(B \otimes C, \rho \otimes \tau)$ is not R-trivial.

Proof. Proposition 2 yields an extension $L_{0} / F_{0}$ such that $\rho_{L_{0}}$ admits an improper similitude $g$ and $B_{L_{0}}$ is not split. Set $L=L_{0}(x, y)$. By Proposition 6,

$$
g \otimes 1 \in \operatorname{Sim}^{+}(B \otimes C, \rho \otimes \tau)(L) \quad \text { and } \quad \theta_{1}(g \otimes 1)=\left[B_{L}\right] \cup(x) \cup(y) .
$$

Since $\left[B_{L_{0}}\right] \neq 0$, taking successive residues for the $x$-adic and the $y$-adic valuations shows that $\theta_{1}(g \otimes 1) \neq 0$. Therefore, $\mathbf{P S i m}^{+}(B \otimes C, \rho \otimes \tau)(L) / R \neq 1$, hence $\operatorname{PSim}^{+}(B \otimes C, \rho \otimes \tau)$ is not $R$-trivial.

\section{InVOLUTIONS OF THE SECOND KIND}

We assume in this section that $(A, \sigma)$ is a central simple algebra with unitary involution over $F$. In this case, the group of similitudes is connected,

$$
\operatorname{Sim}^{+}(A, \sigma)=\operatorname{Sim}(A, \sigma) \quad \text { and } \quad \operatorname{PSim}^{+}(A, \sigma)=\operatorname{PSim}(A, \sigma) .
$$

We denote by $K$ the center of $A$ and write $K=F[X] /\left(X^{2}-\alpha\right)$. We assume the degree of $A$ is even, $\operatorname{deg} A=n=2 m$, and denote by $D(A, \sigma)$ the discriminant algebra of $(A, \sigma)$ (see $[4, \S 10]$ for a definition).

9. Lemma. $D(A, \sigma)$ is split if $(A, \sigma)$ is hyperbolic. 
Proof. The lemma is clear if $A$ is split, for then $\sigma$ is adjoint to a hyperbolic hermitian form $h$ and $[D(A, \sigma)]=(\alpha) \cup(\operatorname{disc} h)$ by $[4,(10.35)]$. The general case is reduced to the case where $A$ is split by scalar extension to the field of functions $L=$ $F\left(R_{K / F}(\mathrm{SB}(A))\right)$ of the Weil transfer of the Severi-Brauer variety of $A$. Indeed, $A \otimes_{F} L$ is split and the scalar extension map $\operatorname{Br}(F) \rightarrow \operatorname{Br}(L)$ is injective by $[9$, Corollary 2.12].

10. Proposition. Suppose $A^{\otimes m}$ is split. The map $\theta_{2}: \operatorname{Sim}(A, \sigma) \rightarrow H^{3}\left(F, \mu_{2}\right)$ defined by

induces a homomorphism

$$
\theta_{2}(g)=(\mu(g)) \cup[D(A, \sigma)]
$$

$$
\Theta_{2}: \operatorname{PSim}(A, \sigma) / R \rightarrow H^{3}\left(F, \mu_{2}\right) .
$$

Moreover, for any $g \in \operatorname{Sim}(A, \sigma)$,

$$
\theta_{2}(g) \cup(\alpha)=0 \quad \text { in } H^{4}\left(F, \mu_{2}\right) .
$$

Proof. In view of the isomorphism (1), it suffices to show that for every finite field extension $L / F$ such that $(A, \sigma) \otimes_{F} L$ is hyperbolic and for every $x \in L^{\times}$,

$$
\left(N_{L / F}(x)\right) \cup[D(A, \sigma)]=0 \quad \text { in } H^{3}\left(F, \mu_{2}\right),
$$

and that for every $\lambda \in K^{\times}$,

$$
\left(N_{K / F}(\lambda)\right) \cup[D(A, \sigma)]=0 \quad \text { in } H^{3}\left(F, \mu_{2}\right) .
$$

As in the proof of Proposition 5, we are reduced by the projection formula to proving that $D(A, \sigma)$ is split by $K$ and by every extension $L / F$ such that $(A, \sigma) \otimes L$ is hyperbolic. The latter assertion follows from the lemma. On the other hand, by $[4,(10.30)]$ and by the hypothesis on $B$ we have

$$
\left[D(A, \sigma)_{K}\right]=\left[\lambda^{m} A\right]=m[A]=0 .
$$

To prove the last part, we use the trace form $T_{\sigma}$ defined as in Section 2,

$$
T_{\sigma}(x)=\operatorname{Trd}_{A}(\sigma(x) x) \quad \text { for } x \in A,
$$

and its restrictions $T_{\sigma}^{+}, T_{\sigma}^{-}$to $\operatorname{Sym}(A, \sigma)$ and $\operatorname{Skew}(A, \sigma)$ respectively. In the case of involutions of unitary type we have

$$
T_{\sigma}=T_{\sigma}^{+} \perp T_{\sigma}^{-}=\langle 1,-\alpha\rangle T_{\sigma}^{+} .
$$

The computation of the Clifford algebra of $T_{\sigma}^{+}$in $[4,(11.17)]$ shows that $T_{\sigma} \in I^{3} F$ and

$$
e_{3}\left(T_{\sigma}\right)=(\alpha) \cup[D(A, \sigma)]
$$

Now, for $g \in \operatorname{Sim}(A, \sigma)$ the map $x \mapsto g x$ is a similitude of $T_{\sigma}$ with multiplier $\mu(g)$, hence $\langle 1,-\mu(g)\rangle T_{\sigma}=0$ in $W F$. Taking the image under $e_{4}$ yields

$$
0=(\mu(g)) \cup e_{3}\left(T_{\sigma}\right)=\theta_{2}(g) \cup(\alpha) .
$$

11. Remarks. (1) If ind $A$ divides $\frac{1}{2} \operatorname{deg} A$, so that $A$ carries a hyperbolic unitary involution $\sigma_{0}$, then $[4,(11.17)]$ and Lemma 9 yield

$$
[D(A, \sigma)]=e_{2}\left(T_{\sigma}^{+}-T_{\sigma_{0}}^{+}\right) .
$$

This observation underlines the analogy between $\theta_{2}$ and the map $\theta_{1}$ of Proposition 5. Note however that no hypothesis on the index of $A$ is required in Proposition 10. 
(2) For $g \in \operatorname{Sim}(A, \sigma)$, the equation $\theta_{2}(g) \cup(\alpha)=0$ implies that $\theta_{2}(g)$ lies in the image of the corestriction map $\operatorname{cor}_{K / F}: H^{3}\left(K, \mu_{2}\right) \rightarrow H^{3}\left(F, \mu_{2}\right)$, by $[4,(30.12)]$. On the other hand, if the characteristic does not divide $m$, Corollary 1.18 of [8] yields an explicit element $\xi \in H^{3}\left(K, \mu_{m}^{\otimes 2}\right)$ such that $\operatorname{cor}_{K / F}(\xi)=\theta_{2}(g)$. In particular, if $m$ is odd it follows that $\theta_{2}=0$.

The following explicit computation yields examples where $\theta_{2} \neq 0$.

12. Proposition. Let $\iota$ be the nontrivial automorphism of $K / F$, and assume

$$
(A, \sigma)=(B, \rho) \otimes_{F}(K, \iota)
$$

for some central simple $F$-algebra with orthogonal involution $(B, \rho)$ of degree $n=$ 2m. Assume $m$ is even. For $g \in \mathrm{GO}^{-}(B, \rho)$ we have $g \otimes 1 \in \operatorname{Sim}(A, \sigma)$ and

$$
\theta_{2}(g \otimes 1)=(\alpha) \cup[B] .
$$

Proof. For $g \in \mathrm{GO}^{-}(B, \rho)$,

$$
\sigma(g \otimes 1) g \otimes 1=\rho(g) g=\mu(g),
$$

so $g \otimes 1 \in \operatorname{Sim}(A, \sigma)$. By $[4,(10.33)]$, we have

$$
[D(A, \sigma)]=m[B]+(\alpha) \cup(\operatorname{disc} \rho) .
$$

Since $m$ is even, the first term on the right side vanishes. The proposition follows by taking the cup-product with $(\mu(g))$, since $[B]=(\mu(g)) \cup(\operatorname{disc} \rho)$ by $[9$, Theorem A] (see also $[4,(13.38)])$.

Remark. If $m$ is odd in Proposition 12, then the definition of $\theta_{2}$ requires the extra hypothesis that $B$ is split by $K$. Computation then shows that $\theta_{2}(g \otimes 1)=0$ for all $g \in \mathrm{GO}^{-}(B, \rho)$, as follows also from Remark 11.2 above.

13. Corollary. Let $r$ be an arbitrary integer. Let $H$ be a quaternion $F$-algebra, $\alpha \in F^{\times}, K=F[X] /\left(X^{2}-\alpha\right)$, and let $\iota$ be the nontrivial automorphism of $K / F$. Assume

$$
(-1) \cup[H]=0 \text { in } H^{3}\left(F, \mu_{2}\right) \quad \text { and } \quad(\alpha) \cup[H] \neq 0 \text { in } H^{3}\left(F, \mu_{2}\right) .
$$

Let $\rho$ be an orthogonal involution on $M_{2 r}(H)$ which admits improper similitudes (see Lemma 1). Then $\operatorname{PSim}\left(M_{2 r}(H) \otimes_{F} K, \rho \otimes \iota\right)$ is not R-trivial.

As in the previous section (see Corollary 8), alternative examples can be constructed from Proposition 2:

14. Corollary. Let $(B, \rho)$ be a central simple algebra of degree 4 with orthogonal involution over a field $F_{0}$. Assume $B$ is not split and $\operatorname{disc} \rho \neq 1$. Let $F=F_{0}(x)$ be the field of rational fractions in one indeterminate over $F_{0}$, let $K=F(\sqrt{x})$ and let $\iota$ be the nontrivial automorphism of $K / F$. The group $\operatorname{PSim}\left(B \otimes_{F_{0}} K, \rho \otimes \iota\right)$ is not R-trivial.

Note that this corollary also follows from [7, Theorem 3]. 


\section{REFERENCES}

[1] G. Berhuy, M. Monsurrò, J.-P. Tignol, The discriminant of a symplectic involution. To appear in Pacific J. of Math.

[2] J.-L. Colliot-Thélène, J.-J. SAnsuc, La R-équivalence sur les tores. Ann. scient. Éc. Norm. Sup., $4^{e}$ série, 10, 175-230 (1997).

[3] P. Gille, Examples of non-rational varieties of adjoint groups. J. Algebra 193, 728-747 (1997).

[4] M.-A. Knus, A.S. Merkurjev, M. Rost, J.-P. Tignol, The Book of Involutions. Amer. Math. Soc. Coll. Pub. 44, AMS, Providence, RI, 1998.

[5] D.W. Lewis, A note on trace forms and involutions of the first kind. Exposition. Math. 15, 265-272 (1997).

[6] Y. Manin, Cubic forms. North-Holland, Amsterdam, 1974.

[7] A. MerkurJev, R-equivalence and rationality problem for semisimple adjoint classical algebraic groups. Pub. Math. IHES 46, 189-213 (1996).

[8] A. Merkurjev, R. Parimala, J.-P. Tignol, Invariants of quasi-trivial tori and the Rost invariant. Preprint.

[9] A. Merkurjev, J.-P. Tignol, The multipliers of similitudes and the Brauer group of homogeneous varieties. J. reine angew. Math. 461, 13-47 (1995).

[10] A. QUÉGUINER, Cohomological invariants of algebras with involution. J. Algebra 194, no 1, 299-330 (1997).

[11] V.E. VoskresenkiĬ, A.A. KlyachKo, Toroidal Fano varieties and root system. Math. USSR Izvestija 24, 221-244 (1985).

Département de mathématiques, École Polytechnique Fédérale de Lausanne, Ch1015 Lausanne, Switzerland

E-mail address: gregory.berhuy@epfl.ch, marina.monsurro@epfl.ch

Institut de Mathématique Pure et Appliquée, Université catholique de Louvain, B1348 Louvain-la-Neuve, Belgium

E-mail address: tignol@math.ucl.ac.be 